\section{HUMAN CANCER-ASSOCIATED FIBROBLAST SUBSETS CAN PREDICT IMMUNE CHECKPOINT RESPONSE IN HEAD AND NECK CANCER PATIENTS}

${ }^{1}$ Diana Graves*, ${ }^{2}$ Aleksandar Obradovic, ${ }^{3}$ Michael Korrer, ${ }^{3} Y u$ Wang, ${ }^{3}$ Sohini Roy, ${ }^{3}$ Yaomin Xu, ${ }^{4} \mathrm{Adam}$ Luginbuhl, ${ }^{4}$ Joseph Curry, ${ }^{3}$ Michael Gibson, ${ }^{3}$ Paula Hurley, ${ }^{5}$ Ravindra Uppaluri, ${ }^{2}$ Charles Drake, ${ }^{2}$ Andrea Califano, ${ }^{3}$ Young Kim. ${ }^{1}$ Vanderbilt University, Antioch, TN, United States; ${ }^{2}$ Columbia University, New York City, NY, United States; ${ }^{3}$ Vanderbilt University Medical Center, Nashville, TN, United States; ${ }^{4}$ Thomas Jefferson University, Philadelphia, PA, United States; ${ }^{5}$ Dana Farber Cancer Institute, Boston, MA, United States

Background Use of anti-PD-1 immune checkpoint inhibitors (ICI) is currently the first line therapy for recurrent/metastatic head and neck squamous cell carcinoma (HNSCC), but critical work remains in identifying factors guiding resistance mechanisms. ${ }^{1}{ }^{2}$ While recent studies have specifically implicated cancer-associated fibroblasts (CAFs) as potential mediators of immunotherapy response, the immunoregulatory role of CAFs in head and neck cancer has not been thoroughly explored. ${ }^{3-5}$ Methods To determine if there are changes in cell populations associated with anti-PD-1 therapy in head and neck cancer patients, we performed high dimensional single-cell RNA sequencing (scRNA-SEQ) from a neoadjuvant trial of 50 advanced-stage head and neck squamous cell carcinoma (HNSCC) patients that were treated with the anti-PD-1 therapy, nivolumab, for the duration of one month. Tumor specimens were analyzed pre- and post-treatment with single-cell RNA sequencing performed on 4 patients as well as bulk RNA sequencing on 40 patients. Matched scRNA-SEQ data was analyzed using the Algorithm for the Reconstruction of Accurate Cellular Networks (ARACNe) and Virtual Inference of Protein-activity by Enriched Regulon (VIPER) bioinformatic analysis platform to determine TME cells that correlated with response and resistance to nivolumab. ${ }^{6}$ For CAF functional studies, surgical tumor specimens were processed and enriched for CAF subtypes, and these were co-cultured with $\mathrm{T}$ cells from peripheral blood and tumor infiltrating lymphocytes.

Results We identified 14 distinct cell types present in HNSCC patients. Of these 14 cell types, the fibroblast subtype showed significant changes in abundance following nivolumab treatment. We identified 5 distinct clusters of cancer-associated fibroblast subsets (HNCAF-0, 1, 2, 3, and 4) of which, two clusters, HNCAF-0 and HNCAF-3 were predictive of patient response to anti-PD-1 therapy. To determine the significance of these CAF subsets' function, we isolated HNCAF-0/3 cells from primary HNSCC tumor specimens and co-cultured with primary human $\mathrm{T}$ cells. Analysis by flow cytometry showed that HNCAF-0/3 reduced TGF $\beta$-dependent PD-1+TIM-3+ exhaustion of $\mathrm{T}$ cells and increased CD103+NKG2A+ resident memory phenotype and cytotoxicity to enhance overall function.

Conclusions To our knowledge, we are the first to characterize CAF heterogeneity within the head and neck TME and show direct immunostimulatory activity of CAFs. Our findings demonstrate the functional importance of CAF subsets in modulating the immunoregulatory milieu of the human HNSCC, and we have identified clinically actionable CAF subtypes that can be used as a biomarker of response and resistance in future clinical trials.

Trial Registration NCT03238365

\section{REFERENCES}

1. Ferris RL, Blumenschein Jr G, Fayette J, Guigay J, Colevas AD, Licitra L, Harrington $\mathrm{K}$, Kasper $\mathrm{S}$, Vokes EE, Even $\mathrm{C}$, et al. Nivolumab for recurrent squamous-cell carcinoma of the head and neck. N Engl J Med 2016;375:1856-1867.
2. Seiwert TY, Burtness B, Mehra R, Weiss J, Berger R, Eder JP, Heath K, McClanahan T, Lunceford J, Gause C, et al. Safety and clinical activity of pembrolizumab for treatment of recurrent or metastatic squamous cell carcinoma of the head and neck (KEYNOTE-012): an open-label, multicentre, phase 1b trial. Lancet Oncol 2016;17:956-965.

3. Dominguez CX, Muller S, Keerthivasan S, Koeppen H, Hung J, Gierke S, Breart B, Foreman O, Bainbridge TW, Castiglioni $A$, et al. Single-cell RNA sequencing reveals stromal evolution into LRRC15(+) myofibroblasts as a determinant of patient response to cancer immunotherapy. Cancer Discov 2020;10:232-253.

4. Feig C, Jones JO, Kraman M, Wells RJ, Deonarine A, Chan DS, Connell CM, Roberts EW, Zhao Q, Caballero OL, et al. Targeting CXCL12 from FAP-expressing carcinoma-associated fibroblasts synergizes with anti-PD-L1 immunotherapy in pancreatic cancer. Proc Natl Acad Sci U S A 2013;110:20212-20217.

5. Kieffer $Y$, Hocine HR, Gentric G, Pelon F, Bernard C, Bourachot B, Lameiras S, Albergante L, Bonneau C, Guyard A, et al. Single-cell analysis reveals fibroblast clusters linked to immunotherapy resistance in cancer. Cancer Discov 2020;10:1330-1351.

6. Obradovic A, Chowdhury N, Haake SM, Ager C, Wang V, Vlahos L, Guo XV, Aggen $\mathrm{DH}$, Rathmell WK, Jonasch $\mathrm{E}$, et al. Single-cell protein activity analysis identifies recurrence-associated renal tumor macrophages. Cell 2021;184:29883005 .

Ethics Approval Patients provided informed consent for this work. All experimental procedures were approved by the Institutional Review Board of Vanderbilt University Medical Center (IRB: 171883).

http://dx.doi.org/10.1136/jitc-2021-SITC2021.903 